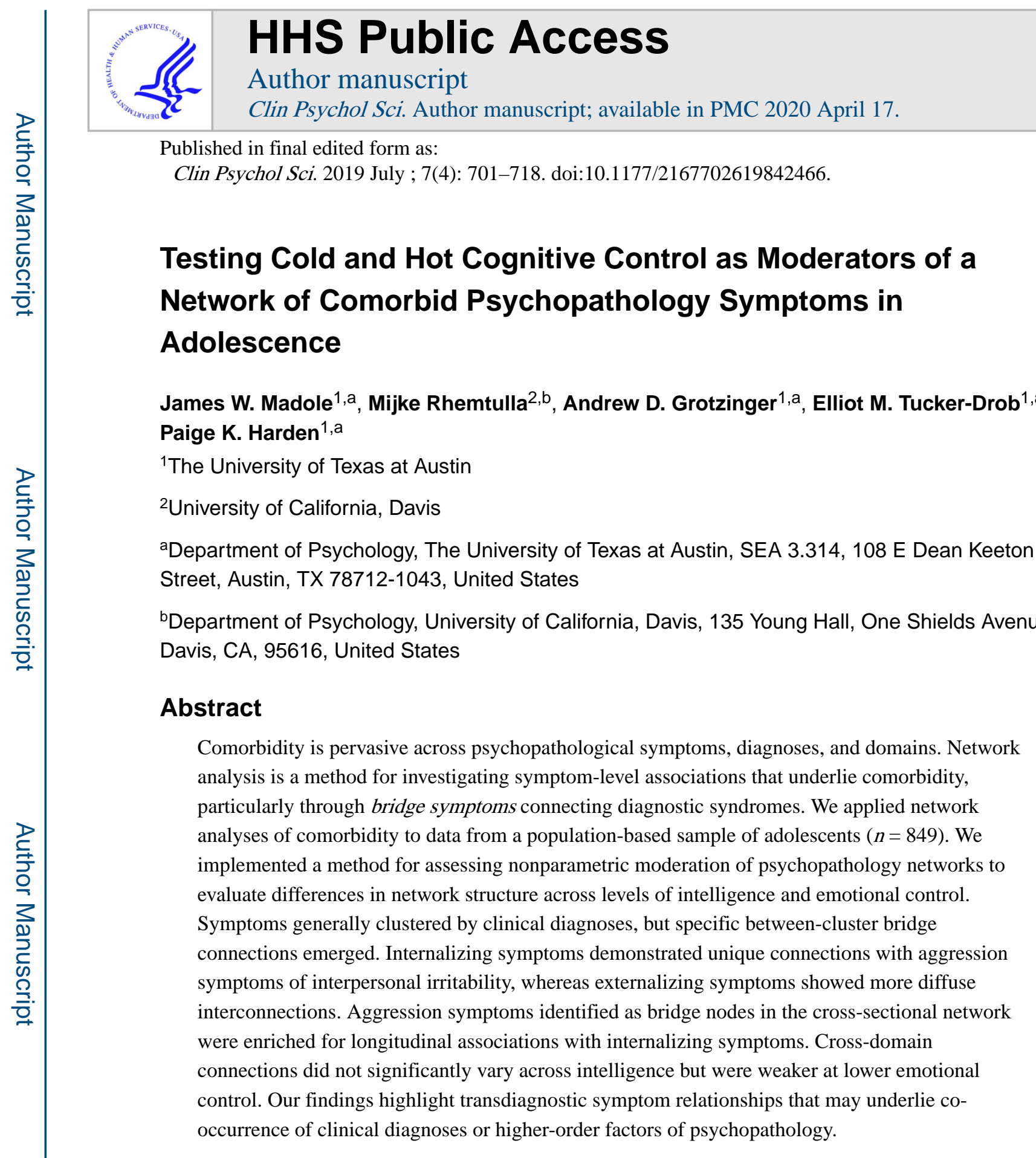

Keywords

psychopathology; comorbidity; network analysis; cognitive control

Correspondence concerning this article should be addressed to James W. Madole, Department of Psychology, The University of Texas at Austin, 108 E Dean Keeton, Stop A8000, Austin, TX 78712-0187. Phone: 512-471-1157, jmadole@utexas.edu. Author Contributions

K. P. Harden, M. Rhemtulla and J.W. Madole developed the study concept and design. J.W. Madole performed the data analysis and interpretation under the supervision of M. Rhemtulla and K.P. Harden. All authors helped in drafting the manuscript and providing critical revisions. All authors approved the final version of the manuscript for submission. 


\section{Network Models of Comorbidity}

Mental disorders are widely comorbid (Hasin \& Kilcoyne, 2012; Kessler, Chiu, Demler, \& Walters, 2005a). Complicating understanding of comorbidity is that clinical diagnoses aggregate over heterogeneous symptom presentations (e.g., Wright et al., 2013; Lindhiem, Bennett, Hipwell, \& Pardini, 2016; Fair, Bathula, Nikolas, \& Nigg, 2012). For example, one study of 3,703 outpatients with major depressive disorder found over 1,000 unique symptom profiles (Fried \& Nesse, 2015a). Given this diversity of symptom presentation, symptomics, defined as a focus on studying individual symptoms of psychopathology, has been championed as a promising avenue for understanding psychiatric comorbidity (Fried et al., 2015; Armour, Fried, \& Olff, 2017). Here, we take a symptomics approach to identifying granular pathways through which mental disorders covary during adolescence, a critical developmental period when more than half of all lifetime cases of psychopathology onset and over a quarter of cases meet for at least one comorbid disorder (Kessler et al., 2005b; Arcelus \& Vostanis, 2005).

Network analysis is a methodological tool for modeling unique relationships between psychopathology symptoms. Although multiple types of network modeling exist (see McNally, 2016 for review), the most commonly employed version of this tool is the concentration network due to its suitability for cross-sectional, correlational data (Epskamp \& Fried, 2017; Epskamp, Waldorp, Mõttus, \& Borsboom, 2018). In concentration networks, the partial pairwise correlations between symptoms are estimated, controlling for all other symptoms (Epkamp \& Fried, 2017). These partial correlations are then typically graphed to allow for both an easily interpretable visualization of the relationships among symptoms, as well as a formal quantification of these relationships using graph theory (Borsboom, 2017; Borsboom \& Cramer, 2013). Results from network analysis have informed a burgeoning conceptualization of psychopathology, network theory, which argues that mental disorders are an upstream reflection of activation patterns among symptoms (Borsboom, 2017; Borsboom \& Cramer, 2013). Within this conceptualization, comorbidity is understood as the occurrence of symptoms from two distinct symptom clusters. Such symptom co-occurrence can arise via bridge symptoms, defined as symptoms from one cluster that have connections with symptoms from another cluster or another clinical disorder (Cramer, Waldorp, van der Maas, \& Borsboom, 2010; Fried et al., 2017).

Previous network analyses investigating the large-scale organization (topology, Constantini $\&$ Perugini, 2016) of psychiatric symptom networks have primarily evaluated (a) how symptoms cluster together and (b) the strength and number of the connections that symptoms display both within and across clusters (Cramer, Waldorp, van der Maas, \& Borsboom, 2010; Boschloo et al., 2015; Boschloo et al., 2016; Goekoop \& Goekoop, 2014). Few studies, however, have used network analysis to address comorbidity across the broad range of mental health disorders (see Fried et al., 2017 for review). Instead, most network analyses of comorbidity have examined only two disorders (Robinaugh, Leblanc, Vuletich, \& McNally, 2014; Ruzzano, Borsboom, \& Geurts, 2014). For example, longitudinal and cross-sectional studies have found symptoms of major depression and generalized anxiety to be densely interconnected, although the precise nature of these interconnections varied by study (Beard et al., 2016; Cramer, Waldorp, van der Maas, \& Borsboom, 2010; Curtiss \& 
Klemanski, 2016; Curtiss, Ito, Takebayashi, \& Hofmann, 2018). Looking across more than two disorders, one study, notable for its size ( $\sim 34,000$ adult patients), found that the network structure of 120 symptoms from 12 disorders generally cohered to DSM-defined clinical boundaries, but that individual symptoms differed substantially in their cross-disorder relationships (Boschloo et al., 2015). For example, all diagnoses were connected via specific symptoms to at least three other diagnoses (Boschloo et al., 2015). Similar results were found in a community sample of 2,175 pre-adolescents ages 10 - 12 (Boschloo et al., 2016). Studies in this vein, which apply network analysis to symptoms of multiple disorders, are particularly important given the growing awareness that widespread comorbidity exists even across domains of psychopathology that appear quite distinct (such as internalizing and externalizing problems) (Caspi \& Moffitt, 2018).

\section{Individual Differences in Network Structure}

The ubiquitous comorbidity among $D S M$ - and $I C D$-defined clinical diagnoses has motivated interest in identifying transdiagnostic risk factors for psychiatric disorders and refining psychiatric nosology accordingly (Clark, Cuthbert, Lewis-Fernández, Narrow, \& Reed, 2017). However, hypothesized transdiagnostic risk factors have not generally been integrated into network analyses of symptom relationships. Here, we propose a novel conceptualization of transdiagnostic risk factors in the context of network theory - as psychological or neurobiological background conditions that strengthen or exacerbate the symptom-tosymptom connections across psychiatric disorders or domains. No previous network analysis study has examined how transdiagnostic symptom relationships might vary as a function of other individual differences (Fried \& Nesse, 2015b; Fried et al., 2015), in part because statistical methods to evaluate moderation of networks by continuously-varying individual differences have not previously been implemented.

We address this methodological and substantive gap by examining individual differences in cognitive control as a moderator of symptom co-activation. Cognitive control is broadly defined as the ability to coordinate thoughts or actions in relation to internal goals (Koechlin, Ody, \& Kouneiher, 2003), and it can be differentiated into "cold" and "hot" forms. Cold cognitive control is defined as the regulatory ability to monitor, direct, and manipulate basic information processing (Zelazo \& Müller, 2002), while hot cognitive control is defined as the regulatory ability to monitor, direct, and manipulate affective processing (Roiser \& Sahakian, 2013). Intelligence test performance is a robust indicator of cold cognitive control (Chuderski \& Nęcka, 2010). Our previous research found that intelligence test performance is highly correlated, both phenotypically and genetically, with a general factor of performance on executive functioning tests, which measure the ability to inhibit responses, shift attention, and update information in working memory (Engelhardt et al., 2016).

Research in a latent variable framework has found that intelligence and executive functioning are negatively associated with a general factor of psychopathology (Caspi et al., 2014; Harden et al., under review; Lahey et al., 2015; Neumann et al., 2016) and with an array of clinically-defined diagnoses (see Snyder, Miyake, \& Hankin, 2015 for review). Meta-analytic work has found that premorbid deficits in cognitive ability, measured by an intelligence test, predicted onset of internalizing, externalizing, and thought disorders 
(David, Zammit, Lewis, Dalman, \& Allebeck, 2008). Focusing on comorbidity specifically, one longitudinal study found that intelligence measured in childhood predicted the cooccurrence of diagnoses in adulthood (Koenen et al., 2009).

Alternatively, some researchers have theorized that cold cognitive deficits might not be the most salient contributors to comorbidity, but rather that failures in hot, or emotional, cognitive control contribute to a cross-cutting liability to experience psychopathology (Carver, Johnson, \& Timpano, 2017; Kret \& Ploeger, 2015). Although emotion regulation is a nuanced construct and may be expressed differentially across psychopathologies (Werner \& Gross, 2010), emotional control has received much attention as a transdiagnostic marker (Kring \& Sloan, 2009). For instance, rash responding to emotion has been tied not only to externalizing disorders like ADHD and conduct disorder, but also to internalizing (i.e., mood and anxiety) disorders in both cross-sectional (Johnson et al., 2017; Johnson, Carver, Joormann, 2013; Marmorstein, 2013) and longitudinal studies (Smith, Guller, \& Zapolski, 2013; Zapolski, Cyders, \& Smith, 2009). Further work has found that problems regulating emotion differentiates clinical groups from healthy controls, but these difficulties are not more pronounced in any particular diagnostic group (Svaldi, Griepenstroh, Tuschen-Caffier, $\&$ Ehring 2012). Meta-analytic work has found strong associations between impulsive responding to emotion and both internalizing and externalizing syndromes (Berg, Latzman, Bliwise, \& Lilienfeld, 2015).

Extant work on cold and hot cognitive control has primarily focused on how these individual differences are associated with or predict psychopathology measured at the diagnostic or higher-order level. In this paper, we take a different line towards approaching how cognitive control influences comorbidity, with a specific focus on how cognitive control moderates the strength of symptom-to-symptom connections across diagnostic boundaries. Specifically, we hypothesized that cold and hot cognitive control may exert transdiagnostic influence by strengthening or weakening connections between symptoms of different domains, such that symptom co-activation is heightened in the face of weak regulatory capacity. For instance, individuals high in negative emotionality (internalizing) might be more likely to hit someone (aggression) if they are ill-equipped with abilities to monitor, direct, or control that emotion. Likewise, individuals who are restless (hyperactivity) might be more likely to disobey at home or school (rule breaking/conduct problems) if they lack the cognitive resources to regulate and focus attention. In this way, poor cognitive control is hypothesized to demarcate a subset of individuals who have co-occurring behavioral and emotional problems that cross diagnostic boundaries.

\section{Goals of the Current Study}

In the current study, we sought to explicate more closely the symptom-level presentation of adolescent psychopathology by estimating its network structure. We further sought to evaluate potential moderators of symptom-level relationships. This is the first study to investigate the network topology of psychopathology in boys and girls during a critical developmental window of adolescence. Further, it is the first study to implement local structural equation models (LOSEM; Briley, Harden, Bates, \& Tucker-Drob, 2015; Hildebrandt, Wilhelm, \& Robitzsch, 2009; Hildebrandt, Luedtke, Robitzsch, Sommer, \& 
Wilhelm, 2016) to assess moderation of psychological networks by individual differences variables. We estimate a series of weighted networks, in which data from each participant are weighted by the participant's distance from a focal value of the moderating variable. By varying the focal value of the moderator across the observed range, we arrive at a nonparametric estimate of how network edges vary as a function of individual differences. We hypothesized that both intelligence, as a proxy for cold cognitive control, and hot, or emotional, cognitive control would moderate connections between symptoms of different domains, such that adolescents with low intelligence and poor emotional control would experience greater comorbidity, as indexed by strength of symptom relationships across different domains.

\section{Method}

Procedures were approved by the university ethics board before data collection commenced. Participants and their parents completed written informed consent procedures.

\section{Participants}

The current sample consists of $n=849$ participants ages $13-20$ years $(M=15.66)$ from the adolescent subsample of the Texas Twin Project (Harden, Tucker-Drob, \& Tackett, 2013), a population-based study of school-aged twins from the Austin and Houston metropolitan areas. Twin pairs were identified from public school rosters and invited to participate in a lab-based study consisting of a battery of psychological assessments. Participants were either currently enrolled in school or had graduated high school within the past three months but had not yet left home for college or full-time work. The sample was approximately gender-balanced (50.7\% male) and was racially diverse: $59.5 \%$ identified as non-Hispanic White, $19.2 \%$ as Hispanic/Latino, $11.4 \%$ as Black/African-American, $2.85 \%$ as Southeast Asian, $1.54 \%$ as East Asian/Pacific Islander, $1.30 \%$ as American Indian/Native American, and $4.16 \%$ identified as other. Participants completed measures of psychopathology, emotional control, and intelligence.

\section{Measures}

Psychopathology.-Adolescent psychopathology was measured using abbreviated versions of three self-report scales: the Achenbach Child Behavior Checklist (CBCL; Achenbach \& Edelbrock, 1991); the DSM-IV symptom count scales of the Conners 3 rating scales (Conners, Pitkanen, \& Rzepa, 2011), and the neuroticism subscale of the Big Five Inventory (BFI-N; John \& Srivastava, 1999). The scales were selected to cover a broad range of typical psychopathological problems in adolescence: depression (e.g., "There is very little that I enjoy"), measured with the BFI-N and CBCL; anxiety (e.g., "I am nervous or tense"), measured with the BFI-N and CBCL; inattention (e.g., "I have trouble concentrating/paying attention"), measured with the CBCL and Conners; hyperactivity (e.g., "I am restless"), measured with the CBCL and Conners; learning problems (e.g., "I learn more slowly than other kids my age"), measured with the Conners; rule breaking and conduct problems (e.g., "I steal at home"), measured with the CBCL and Conners; and aggression (e.g., "I break things (when angry/upset)"), measured with the CBCL and Conners. More severe and 
infrequent forms of adolescent psychopathology, including schizophrenia, bipolar disorder, and other thought disorders, were not considered in these analyses.

Items on the BFI-N ranged from 1 ("Strongly Disagree") to 5 ("Strongly Agree"); items on the CBCL ranged from 0 ("Not True") to 2 ("Very True or Often True"); and items on the Conners ranged from 0 ("Not True at All") to 3 ("Very Much True"). All scales have demonstrated good validity: Neuroticism has demonstrated strong relationships with internalizing psychopathologies in adolescents $(r=.98)$ (Griffith et al., 2010) and has shown substantial genetic overlap with internalizing symptoms (Hettema et al., 2006); the CBCL has demonstrated excellent psychometric properties, including concurrent validity with DSM diagnoses in children and adolescents (Nakamura, Ebesutani, Bernstein, \& Chorpita, 2008; Ebesutani et al., 2010); and the Conners has demonstrated adequate convergent validity with similar measures in children and early adolescents (Erford, 1995).

Emotional control.-Emotional control was measured using the urgency subscale of the UPPS Impulsive Behavior Scale (e.g., "When I am upset I often act without thinking"). Participants responded to items on a scale of 1 ("Disagree Strongly") to 4 ("Agree Strongly"). Items were reverse scored such that greater scores indicated less urgency, or greater emotional control. The psychometric properties of the UPPS are well-established (Whiteside \& Lynam,2001; Whiteside, Lynam, Miller \& Reynolds, 2005). The UPPSUrgency subscale has demonstrated significant correlations with self-report measures of emotion dysregulation (Fossati, Gratz, Maffei, \& Borroni, 2014) and has achieved good reliability and validity as a measure of impulsive responding to emotion (Cyders \& Smith, 2007; Cyders \& Smith, 2010).

Intelligence.-Intelligence was assessed using the Wechsler Abbreviated Scale of Intelligence-II (WASI-II; Wechsler, 2011). The WASI-II measures two domains of intelligence: visuospatial reasoning, consisting of Block Design and Matrix Reasoning subtests, and verbal ability, consisting of Vocabulary and Similarities subtests. Scores from the four subtests are individually normed and combined to form Full-Scale IQ (FSIQ). FSIQ in the current sample reflects population norms $(M=103.54, S D=13.19)$. The WASI has demonstrated significant associations with cognitive control measures in children and adolescents (Andrews-Hanna et al., 2011; Solomon, Ozonoff, Cummings, \& Carter, 2008).

\section{Analyses}

Our analytic plan encompasses three broad aims: (1) to elucidate the cross-sectional network structure of adolescent psychopathology by investigating clustering of symptoms in the network and the nature of the connections within and between those clusters; (2) to evaluate how this network structure differs across levels of cold and hot cognitive control by implementing a novel method of nonparametric moderation; (3) to examine whether bridge symptoms identified in cross-sectional networks uniquely predict future psychopathology in a longitudinal follow-up analysis.

$\mathrm{R}$ and Mplus scripts for all analyses are included as supplementary files. Prior to analyzing the data, all items were coded such that higher scores reflect greater severity of psychopathology. All psychopathology items, with two exceptions described below, were 
treated as ordinal variables, gender and ethnicity were scored as dichotomous variables (male/female; minority/non-minority status), and age was treated as continuous. Both intelligence and emotional control were $z$-scored prior to analyses and were treated as continuous variables.

Less than $.1 \%$ of the data were missing. Participants with more than $20 \%$ missing data ( $n=$ 7) were removed from analyses. Single PMM-based imputation was then conducted on the remaining cases using the mice package in $R$ (van Buuren \& Groothuis-Oudshoorn, 2011). Single imputation is considered an appropriate technique when less than $5 \%$ of data are missing (Graham, 2009).

103 items were included in our analyses, 61 of which were treated as indicative of unique symptoms. The 42 remaining items were combined to form 15 composite variables according to two justifications. First, based on the wording of the prompt, items that were theorized to tap the same symptom (e.g., BFI-N item 4: "is depressed, blue" and CBCL item 44: "I am unhappy, sad, or depressed") were summed in order to reduce topological overlap (Fried, Epskamp, Nesse, Tuerlinckx, \& Borsboom, 2016). Second, 11 items endorsed by less than $10 \%$ of the sample were combined with related items in order to ameliorate the bias created by their skew. Two of these - a property damage variable created by summing 7 ordinal items and a physical aggression variable created from another 7 ordinal items - were treated as continuous. Truancy, a sum of 3 ordinal items, was treated as an ordinal variable with 7 levels. One item ("I get into trouble with the police") was dropped from the analyses due to low endorsement rate. All final variables were endorsed by at least $15 \%$ of the sample.

Network of adolescent psychopathology.-Psychopathology networks consist of nodes, representing individual symptoms, and edges, representing connections among symptoms. In concentration networks, edges represent conditional pairwise linear associations between two variables controlling for all other variables in the network.

Estimation \& visualization.: A full description of the network estimation and visualization method are attached as Supplementary Method S1. Briefly, we estimated the symptom network from the observed polyserial correlation matrix using the EBICglasso function in the qgraph package in $R$ (Epskamp, Cramer, Waldorp, Schmittmann, \& Borsboom, 2012). To avoid overfitting, coefficients (i.e., edges) are regularized using an $l_{1}$-penalty, which shrinks weak edges to exactly zero. Networks estimated using the EBICglasso approach typically have high sensitivity (the edges that are estimated are very likely to be non-zero) but low specificity (edges that are estimated to be zero may or may not truly be zero) (Foygel \& Drton, 2010). The network structure was visualized using the qgraph package in $R$ (Epskamp, Cramer, Waldorp, Schmittmann, \& Borsboom, 2012). The thickness of an edge represents the strength of the association such that thicker edges indicate stronger associations. Green edges reflect positive associations, whereas red edges reflect negative associations.

Cluster analyses.: The spinglass algorithm, employed using the igraph package in $R$ (Csardi \& Nepusz, 2006), was used to identify communities of nodes in the graph; 
communities are defined as a set of nodes with relatively many edges inside the community and fewer edges connecting the community to the rest of the graph (Reichardt \& Bornholdt, 2006). Because the algorithm is prone to variability in grouping, it was run 1000 times. The number of clusters identified ranged from $5-8$, with 6 being the median number of clusters identified. Of the 457 runs that identified 6 clusters, we used the most frequently derived clustering arrangement $(n=239)$ to determine group membership. Results are discussed in terms of connections within and between the clusters (i.e., domains) identified using this algorithm.

Network moderation.: We used local structural equation modeling (LOSEM) to assess intelligence and emotional control as continuous moderators of the unique association between each pair of symptoms (Briley, Harden, Bates, \& Tucker-Drob, 2015; Hildebrandt, Wilhelm, \& Robitzsch, 2009; Hildebrandt, Luedtke, Robitzsch, Sommer, \& Wilhelm, 2016). LOSEM uses kernel estimation techniques to generate nonparametric estimates of differences in structural equation model parameters across levels of a measured moderator. In combination with the capability of structural equation modeling to produce a saturated model representing the full heterogeneous correlation matrix among all pairs of symptoms, we used LOSEM to generate a series of correlation matrices that are generated across the range of observed moderator values. Each correlation matrix was derived by re-weighting the sample data, with sample weights reflecting the proximity of each individual's score on the moderator to the focal value being tested for that particular iteration.

Correlation matrices were computed across -2 to $2 S D s$ of intelligence and emotional control at increments of .1 $S D$ units, resulting in 41 correlation matrices for each moderator. Observed moderator values were included in each LOSEM-weighted correlation matrix and resulting network, to control for the main effect of the moderator on each symptom. This prevented estimates of the moderation of symptom associations from being biased by differences in rates of symptom endorsement across levels of the moderator. The resulting weighted polyserial correlation matrices were estimated in Mplus and analyzed in R. For instances in which no positive definite matrix was produced ${ }^{1}$, we used the nearPD function in the Matrix package in $R$ (Bates \& Maechler, 2016) to find the nearest positive definite matrix for any observed non-positive definite matrix. Networks that included the respective moderator as a node were then estimated from each polyserial matrix using the glasso function in $R$ (Friedman, Hastie, \& Tibshirani, 2014). This function uses the same regularization procedure as described above, but allows the analyst to fix the $I_{1}$-penalty, rather than choosing it anew using EBIC model selection for each network. For each LOSEM-weighted matrix, we fixed the $l_{1}$-penalty to be equal to the optimal EBIC-derived value obtained in the unweighted network that included the moderator as a node ( $\lambda=.122$ for both). Variation in edge weights thus reflects interpretable differences rather than potential artifacts of the regularization process.

In order to facilitate comparisons of symptom covariance differences across levels of the moderators, we constrained cluster membership in the LOSEM-weighted matrices to be

${ }^{1}$ Polychoric correlation matrices are often non-positive definite because the entire correlation matrix is not estimated at one time, and this problem appears to be compounded when using weighted polychoric matrices (Rigdon, 1997). 
equal to the unweighted graph. To quantify differences in network structure across the range of each moderator, we calculated an average between-cluster edge weight (i.e., regularized, partial correlation coefficient; $\left.M_{I(\mathrm{BC})}\right)$ for each network across the range of the moderator. $M_{r(\mathrm{BC})}$ was chosen a priori, as we predicted that we would observe larger positive edges between symptoms of different psychopathological domains at lower levels of cognitive control. That is, we would expect $M_{I(\mathrm{BC})}$ to decrease as the level of each moderator increases. $M_{r(\mathrm{BC})}$ was calculated by summing edge weights connecting nodes from different clusters and dividing by the total number of possible between-cluster connections. Thus, it can be interpreted as the average positive unique association across symptom domains. $M_{I(\mathrm{BC})}$ is particularly useful in that it accounts for negative coefficients, which, based on how our data were scored, would reflect the absence of one symptom in the presence of another, and thus would not be indicative of comorbidity.

To test whether the observed variation in $M_{r(\mathrm{BC})}$ was significantly different from what would be observed by chance, we ran a permutation test in which intelligence and emotional control were randomly reordered across participants, resulting in 1000 permuted datasets. Each case's observed value for intelligence and emotional control were retained as a variable in the permuted datasets so that the main effect of the moderator on symptom relationships did not vary as a function of the random reordering of the moderator. We then ran each of the $(k=1000)$ permuted datasets through the LOSEM procedure and calculated the $M_{I(\mathrm{BC})}$ at each value of moderator $(m=41)$, resulting in an empirical null distribution of $k \times m$ values of $M_{I(\mathrm{BC})}$. Observed $M_{I(\mathrm{BC})}$ values between $-1.5-+1.5 S D$ s of the moderator were evaluated as significant if they were smaller than the bottom $2.5 \%$-ile or larger than the top $97.5 \%$-ile of the empirical null distribution.

Longitudinal follow-up.: To investigate the direction of associations between bridge symptoms identified in the cross-sectional, unmoderated network analysis, we estimated a longitudinal network of symptoms in a subsample of Texas Twin Project participants ( $n=$ 218) who completed two waves of assessment. Supplementary Table S2 contains descriptive statistics for the longitudinal subsample. Each symptom in the longitudinal network was represented by two nodes - one for each time point. Analyses focus on edges between Wave 1 and Wave 2 nodes, which represent the predictive associations between one symptom and future levels of another symptom, above and beyond all other symptoms in the network. We focus primarily on the percentage of connections (i.e., number of observed positive connections / number of potential positive connections) between bridge symptoms and disparate domains of psychopathology.

\section{Results}

\section{Reliability Analyses}

Before estimating the network in the full sample, we conducted a split-half reliability analysis to establish confidence in the parameters generated by the network estimation process. Participants in each twin pair were arbitrarily assigned to Twin $1(n=417)$ or Twin $2(n=425)^{2}$, and within each of these subgroups, we estimated correlation matrices in Mplus and estimated networks using EBICglasso, as described in the previous section. We 
then correlated the edge weights across the two twin networks to index network similarity (Borsboom et al., 2017; Fried et al., 2018; Rhemtulla et al., 2016). We focus here on edge weights as our analyses primarily concern the magnitude and number of these connections. We demonstrate good stability of our strength index, using the bootnet function (Epskamp, Borsboom, \& Fried, 2017), in Supplementary Figure S3.

The two correlation matrices, from which Twin 1 and Twin 2 networks were estimated, correlated at .848. We derived an optimal penalization parameter to fix across networks by first estimating each network individually using the EBICglasso function. We re-estimated the networks using the glasso function with the penalty parameter fixed to the average value of the two individual networks $(\lambda=.26)$. The edge weights from these two networks correlated at .782, which we interpret as adequate reliability given the upper bound established by the correlation between the polyserial matrices. To ensure that split-half and full sample networks did not diverge as a function of the penalty parameter, we estimated the twin networks again using the penalty derived from the full sample $(\lambda=.111)$. The edge weight correlation remained adequate $(r=.740)$.

\section{General Network Structure of Adolescent Psychopathology}

Figure 1 depicts the estimated network, where nodes represent psychopathology symptoms and edges represent the unique associations among them (see Supplementary Table S4 for node reference). Six clusters were identified using the method described above, displaying general coherence with clinical syndromes. As shown in Supplementary Table S4, only five nodes out of 76 were found to cluster with groups different from their self-report subscale.

Age, gender, and ethnicity were omitted from clustering analyses, and connections between these correlates and other nodes are not reported.

We focus our analyses on the 2,850 potential connections (i.e., 76*75/2) among nodes. Figure 2 shows the number and relative average strength of the connections from all nodes to each of the six domains, as well as a summary of the total between-cluster connections for each node. Connection strength was calculated by averaging all edge weights from an individual node to all nodes of a particular domain. Nodes that have an average edge weight with a given domain at or above the $75^{\text {th }}$ percentile of all average edge weights are characterized as "relatively strong". Strength of total between-cluster connections (i.e., an individual node's connections with all other nodes outside of its cluster) was calculated by averaging the edge weights from an individual node to every node not within its cluster. Nodes with the most and strongest connections to each domain and to all between-cluster domains are detailed in Supplementary Table S5. Reliability of the ordering of these nodes was established by conducting a bridge centrality analysis, which calculates how central (i.e., number of connections, proximity in graphical space) nodes are to clusters outside of their own, using the networktools package in $R$ (Jones, 2018). Supplementary Figure S6 details the results from this analysis, which demonstrates adequate consistency with our results. Supplementary Table S7 contains the number and relative strength of connections

\footnotetext{
${ }^{2}$ Sibling 3 was grouped with Twin 2 for those families with triplets.
} 
within and between clusters, in which all present edge weights were rank-ordered to determine percentile cutoffs.

Overall, externalizing symptom domains (rule breaking/conduct problems, aggression, and hyperactivity) were consistently interconnected, with multiples nodes from each externalizing domain showing relatively strong average connections with all other externalizing domains. Rather than being broadly interconnected with externalizing, learning problems showed more specific connections with inattention symptoms. Internalizing symptoms showed the weakest and sparsest connections with other symptom domains. Interestingly, the nodes connecting internalizing and externalizing were specific to aggression, in particular, experiences of interpersonal irritability (e.g., "people make me angry"). In the following sections, we briefly summarize connections involving each domain.

Internalizing.-Internalizing nodes $(p=17)$ displayed $4-12$ connections with one another, and $2-13$ connections (out of 59 possible) with nodes of other domains. Two internalizing nodes ("I feel confused or in a fog" and "[I] can be moody") displayed relatively strong average between-cluster connections. Considering nodes from other domains, 20 out of 59 displayed no connections with the internalizing domain, whereas only 3 nodes from other domains displayed relatively strong average connections with internalizing nodes. These nodes with strong average connections to internalizing ("People make me angry"; "I am suspicious"; and "I scream a lot") were all from the aggression domain. Longitudinal prediction of these three symptoms in connecting internalizing and aggression was addressed in a follow-up analysis below.

Learning Problems.-Learning problems nodes $(p=7)$ displayed between $3-6$ connections with one another, and between $2-10$ connections (out of 69 possible) with nodes of other domains. Two nodes ("I need help doing my homework" and "I forget things that I have learned") displayed relatively strong average between-cluster connections. Considering nodes from other domains, 42 out of 69 displayed no connections with the learning problems domain, whereas only 5 nodes from other domains displayed relatively strong average connections with learning problems nodes. These nodes ("I have trouble keeping my mind on what people are saying to me"; "I am behind in my schoolwork"; "I have trouble concentrating/paying attention"; "I don't like doing things that make me think hard"; and "I have trouble following instructions") were all from the inattention domain.

Hyperactivity.-Hyperactivity nodes $(p=14)$ displayed between $5-10$ connections with one another, and between $2-17$ connections (out of 62 possible) with nodes of other domains. Two of these nodes ("I interrupt other people" and "I get out of my seat when I am not supposed to") displayed relatively strong average between-cluster connections. Considering nodes from other domains, 18 out of 62 displayed no connections with the hyperactivity domain, whereas 7 nodes from other domains displayed relatively strong average connections with hyperactivity nodes. Three of these nodes came from aggression, two from rule breaking/conduct problems, and two from inattention. 
Rule Breaking/Conduct Problems.-Rule breaking/conduct problems nodes ( $p=10$ ) displayed between $4-9$ connections with one another, and between $5-14$ connections (out of 66 possible) with nodes of other domains. Three of these nodes ("I lie or cheat (to get out of doing stuff)"; "I act without stopping to think"; and "I disobey at school") displayed relatively strong average between-cluster connections. Considering nodes from other domains, 19 out of 66 displayed no connections with the rule breaking/conduct problems domain, whereas 11 nodes from other domains displayed relatively strong average connections with rule breaking/conduct problems nodes. Six of these nodes came from aggression, three came from hyperactivity, and two came from inattention.

Inattention.-Inattention nodes $(p=12)$ displayed between $4-10$ connections with one another, and between $0-15$ connections (out of 64 possible) with nodes of other domains. Four of the inattention nodes displayed relatively strong average between-cluster connections. 17 out of 64 nodes from other domains displayed no connections with the inattention domain. Six nodes from other domains displayed relatively strong average connections with inattention nodes. Three of these nodes came from learning problems ("I learn more slowly than other kids my age"; "I need help doing my homework"; and "I forget things that I have learned"), one from hyperactivity ("I have trouble sitting still"), and one node each from rule breaking/conduct problems ("I disobey at school") and internalizing ("I feel confused or in a fog").

Aggression.-Aggression nodes $(p=16)$ displayed between $4-11$ connections with one another, and between $5-14$ connections (out of 60 possible) with nodes of other domains.

Six of the aggression nodes displayed relatively strong average between-cluster connections. Considering nodes from other domains, 15 out of 60 displayed no connections with the aggression domain, whereas 9 nodes from other domains displayed relatively strong average connections with aggression. Four nodes came from rule breaking/conduct problems, 3 came from hyperactivity, and one node each came from inattention ("I lose stuff that I need") and internalizing ("[I] can be moody").

Total Between-Cluster Connections.-Nineteen of the 76 nodes in total displayed relatively strong average between-cluster connections. Clusters with the highest number of nodes with strong average between-cluster connections were aggression $(p=6)$, inattention $(p=4)$, and rule breaking/conduct problems $(p=3)$. Four of the six aggression nodes were related to interpersonal difficulties (e.g., "I try to annoy other people"). Two nodes displayed strong average connections for hyperactivity ("I interrupt other people" and "I get out of my seat when I'm not supposed to"), learning problems ("I need help doing my homework" and "I forget things that I have learned"), and internalizing ("I feel confused or in a fog" and "[I] can be moody").

\section{Moderated Network Structure across Cold and Hot Cognitive Control}

Before assessing differences in network structure across the range of cold and hot cognitive control, we first estimated unmoderated networks that included each moderator as a node. We determined the centrality, indexed by the number of connections that each node displays, of each moderator to get a global sense of the relevance of each moderator variable to the 
network. Intelligence was the most central node to its network. Emotional control was the $11^{\text {th }}$ most central node to its network.

Moderation was evaluated in terms of average between-cluster edge weight $\left(M_{r(\mathrm{BC})}\right)$. This metric, which represents the average edge weight between a given node and all nodes of different domains (i.e., $n=2,371$ potential between-cluster connections) was chosen to provide a global indication of symptom co-occurrence across different domains. To quantify differences in $M_{I(\mathrm{BC})}$ across moderated networks, we created an empirical null distribution from permuted $M_{I(\mathrm{BC})}$ values and evaluated significance based on observed $M_{I(\mathrm{BC})}$ values that fell in the upper or lower $2.5 \%$ of the empirical null distribution. Figures $3 \mathrm{a}$ and $3 \mathrm{~b}$ illustrate the observed $M_{I(\mathrm{BC})}$ values in relation to the empirical null distribution.

Across the range of intelligence, none of the observed $M_{I(\mathrm{BC})}$ values fell outside of the upper or lower $2.5 \%$ of the empirical null distribution, although there was a nonsignificant pattern of greater comorbidity (higher $M_{r(\mathrm{BC})}$ ) at lower intelligence. In contrast to the intelligence analysis, the emotional control analysis revealed a significant pattern of less comorbidity (lower $M_{r(\mathrm{BC})}$ ) between -1.5 and -.6 SDs of emotional control (empirical $p<.005-$ empirical $p<.025)$, indicating that disparate domains of psychopathology are, on average, more weakly connected at lower levels of emotional control. Supplementary Table S8 contains the unique between-cluster symptom connections that display the greatest variance across the range of each moderator.

To further probe the finding that between-cluster symptoms are more weakly connected at low levels of emotional control, we ran exploratory post-hoc analyses to investigate whether connections between specific domains were driving this finding. Supplementary Figures S9 \& S10 illustrate the between-cluster connections between (a) internalizing and aggression symptoms and (b) externalizing domains (aggression, rule breaking/conduct problems, hyperactivity), respectively. We found that the average internalizing to aggression connection was significantly lower than chance at both the lower $(-1.5--1.1 S D \mathrm{~s})$ and upper $(0.7-1.5 S D \mathrm{~s})$ tails of emotional control, indicating that individuals with strong emotional control as well as individuals with poor emotional control demonstrate significantly weak connections between internalizing and aggression symptoms. We found that the average connection between externalizing domains was significantly lower than chance from $1.4-1.5 S D$ s of emotional control (empirical $p<.025$ ).

\section{Longitudinal Prediction of Internalizing-Aggression Bridge Symptoms}

To determine whether the three aggression nodes identified as bridge nodes in the crosssectional network prospectively predicted, or were prospectively predicted by, internalizing symptoms, we created two sampling distributions ( $k=10,000$ each): (1) the percentage of connections between Wave 1 internalizing nodes and three randomly sampled Wave 2 aggression nodes, and (2) the percentage of connections between three randomly sampled Wave 1 aggression nodes and Wave 2 internalizing nodes.

Internalizing at Wave 1 Predicting Bridge-Aggression at Wave 2-Internalizing nodes at Wave 1 displayed $7.8 \%$ of potential connections with the three bridge-aggression nodes at Wave 2, but displayed only $1.4 \%$ of potential connections with non-bridge- 
aggression nodes at Wave 2. Observed connections from Wave 1 internalizing to Wave 2 bridge-aggression nodes were significantly greater than connections between Wave 1 internalizing nodes and randomly sampled aggression nodes at Wave 2 (empirical $p<.001$; empirical distribution range: $0.0-7.8 \%$ ). Supplementary Figure S11 displays the observed percentage of internalizing to bridge-aggression connections within the empirical cumulative distribution function of the percentage of connections in randomly sampled data.

Bridge-Aggression at Wave 1 Predicting Internalizing at Wave 2-Bridgeaggression nodes at Wave 1 displayed $11.8 \%$ of potential connections with internalizing nodes at Wave 2, whereas non-bridge-aggression nodes at Wave 1 displayed only $3.2 \%$ of potential connections with internalizing nodes at Wave 2. Observed connections from Wave 1 bridge-aggression to Wave 2 internalizing nodes were significantly greater than connections between randomly sampled aggression nodes at Wave 1 and internalizing nodes at Wave 2 (empirical $p<.02$; empirical distribution range: $0.0-13.7 \%$ ). Supplementary Figure S12 displays the observed percentage of bridge-aggression to internalizing connections within the empirical cumulative distribution function of the percentage of connections in randomly sampled data.

\section{Discussion}

To our knowledge, this is the first study to examine transdiagnostic psychopathology symptom covariation in adolescence using a network of partial, regularized correlations. This study was also innovative in its application of a method for assessing the moderating role of individual differences in cognitive control on symptom-level comorbidity. We found that symptoms in the network generally clustered according to clinically-defined boundaries, with clustering defined as many and relatively strong interconnections.

Looking across clusters, we found specific patterns of association between different domains. Internalizing symptoms connected strongly to just three symptoms of aggression, all measuring interpersonal irritability. Longitudinal follow-up demonstrated the prognostic value of these symptoms in connecting future internalizing and aggression problems. That is, symptoms of interpersonal irritability were significantly predictive of, and predicted by, internalizing symptoms relative to other aggression symptoms. This finding is consistent with recent work that found irritability and interpersonal difficulty to be amongst the most salient bridge symptoms connecting these domains (Rouquette et al., 2018). That frustration and displeasure with other people are bridge symptoms for the internalizing and externalizing domains complements previous work showing that reactive, but not proactive, aggression is related to internalizing symptoms in adolescents (Fite, Rubens, Preddy, Raine, \& Pardini, 2014; Fite, Stoppelbein, \& Greening, 2009; Fite, Rathert, Stoppelbein, \& Greening, 2012). Learning problems displayed similar between-cluster specificity, demonstrating strong average connections with only five symptoms of inattention. The specificity of this relationship is consistent with literature demonstrating that teacherreported attention problems predict poor academic performance (Lundervold, Bøe, \& Lundervold, 2017) more strongly than other common forms of child and adolescent psychopathology (Sijtsema, Verboom, Penninx, Verhulst, \& Ormel, 2014). Externalizing domains (rule breaking/conduct problems, aggression, and hyperactivity) showed more 
disperse interconnectedness, consistent with factor-analytic work that has demonstrated the non-specificity of externalizing symptoms to diagnostic categories (Krueger, Markon, Patrick, \& Iacono, 2005; Krueger et al., 2002).

Nineteen symptoms emerged as having relatively strong, average connections with all other cross-cluster symptoms. We interpret these as transdiagnostic bridge symptoms, or symptoms that might be important unifiers of psychopathology in adolescence. Six of these symptoms were again relevant to interpersonal irritability, a finding that is consistent with interpersonal theories of developmental psychopathology, which posit that diverse psychopathologies arise from conflict between interpersonal difficulties and basic needs for kinship (Rudolph, Lansford, \& Rodkin, 2016). Particularly given that our longitudinal analyses demonstrated that symptoms of interpersonal irritability predicted future internalizing symptoms (and vice versa), it is intriguing to speculate whether psychological interventions targeting interpersonal irritability would decrease rates of comorbidity between internalizing and externalizing psychopathology.

Considering the contribution of cognitive control to symptom-level comorbidity, intelligence, when added as a node in the network, demonstrated the greatest number of connections to all other nodes in the network. This supports the well-established relevance of cognitive ability to transdiagnostic psychopathology (Caspi \& Moffitt, 2018; Snyder, Miyake, \& Hankin, 2015). Our finding extends this work by highlighting that cognitive ability may be centrally important to psychiatric comorbidity via its pervasive connections to specific symptoms. However, we found that intelligence was not significantly related to the average strength of between-cluster connections.

In contrast, we found that emotional control was less central to the network but displayed more relevance in moderation analyses than intelligence. Contrary to our hypothesis, between-cluster symptom connections were significantly weaker at lower levels of emotional control. Given the counter-intuitiveness of this finding, we conducted exploratory post-hoc analyses to probe connections between specific domains. Analyses of the average connection between internalizing and aggression domains and between externalizing domains were more consistent with the hypothesized trend of greater between-cluster symptom co-activation at lower levels of emotional control. These were post-hoc exploratory analyses that warrant replication, and they do not account for the global trend observed across all domains of psychopathology. Taken together, our findings do not support the hypothesis that cognitive control moderates the cooccurrence of symptoms across domains of psychopathology. However, the method we introduce for generating LOSEM-weighted networks to analyze moderation of psychological networks can be productively applied to examine other potential moderators, both experimentally manipulated (e.g., type of psychological treatment) and naturally occurring (e.g., treatment adherence).

Our study has four major limitations. First, although a prominent version of "network theory", distinct from but informed by network analysis, proposes that causal relationships between symptoms drive and sustain psychopathological networks (Borsboom, 2017; Borsboom \& Cramer, 2013), our data were primarily cross-sectional correlations that cannot be used to ascertain causal relationships. Indeed, the measurement of causal processes 
within an idiographic system are most proximally captured using high density sampling methods (e.g., ecological momentary assessment) (Fisher, 2015; Hofmann, Curtiss, \& McNally, 2016). Cross-sectional data or between-person longitudinal data cannot comment on such processes. Rather, such data has the capacity to draw nomothetic inferences about symptom covariance and the factors that impinge on that covariance in a network. Second, data were self-report. Child and adolescent behavior is subject to contextual variability, and the use of multiple reporters can help to increase the accuracy and coverage of emotional and behavioral problems (Hunsley \& Mash, 2007; Dirks, De Los Reyes, Briggs-Gowan, Cella, \& Wakschlag, 2012). Network analyses of child and adolescent samples may benefit from using diverse assessment instruments to capture symptom-level presentation more precisely in these populations. Third, although our data cover the most common forms of psychopathology in adolescence (Michaud \& Fombonne, 2005; Achenbach, 1966; Achenbach \& Edelbrock, 1983), symptoms from rarer forms of psychopathology, such as schizophrenia, bipolar disorder, or autism spectrum disorders, were not assessed. Future studies would benefit from a more comprehensive scope of transdiagnostic psychopathology symptoms in adolescence. Fourth, cross-cluster comorbidity was quantified using a single, global metric that is opaque regarding which disorders, and which symptoms within those disorders, most robustly drive the metric.

In conclusion, our study extends work on psychiatric comorbidity by highlighting unique symptom relationships that potentially drive the co-occurrence of distinct domains of psychopathology. We highlight a number of bridge symptoms that may be salient conduits of comorbidity in adolescence. Particularly, we illustrate the specificity of symptoms of interpersonal irritability in uniting internalizing and aggression symptoms over time. Further, we demonstrate that intelligence, as a proxy for cold regulatory capacities, is pervasively associated with psychopathological symptoms, but does not impact liability for comorbid symptom to symptom relationships in a network. In contrast, emotion-based regulatory ability is associated with the strength of symptom comorbidity, though its association is dependent on the specific domains in question. This study highlights interpersonal irritability as promising intervention targets for adolescents dealing with both internalizing and externalizing problems.

\section{Supplementary Material}

Refer to Web version on PubMed Central for supplementary material.

\section{Acknowledgements}

Data from the Texas Twin Project were collected and managed using Research Electronic Data Capture (REDCap) hosted at The University of Texas at Austin (UT-Austin). During the time that this article was prepared, KPH and EMTD were supported by National Institutes of Health (NIH) grants R01HD083613, R21HD081437, and R21AA023322. KPH and EMTD are Jacobs Foundation Research Fellows. The Population Research Center at the University of Texas is supported by NIH grant R24HD042849.

\section{References}

Achenbach TM (1966). The classification of children's psychiatric symptoms: a factor-analytic study. Psychological Monographs: General and Applied, 80, 1-37. doi:10.1037/h0093906 
Achenbach TM, \& Edelbrock CS (1983). Manual for the child behavior checklist: and revised child behavior profile. Burlington: University of Vermont, Department of Psychiatry.

Achenbach TM, \& Edelbrock C (1991). Child behavior checklist. Burlington (Vt), 7.

Andrews-Hanna JR, Seghete KLM, Claus ED, Burgess GC, Ruzic L, \& Banich MT (2011). Cognitive control in adolescence: neural underpinnings and relation to self-report behaviors. PloS One, 6 , e21598. doi:10.1371/journal.pone.0021598 [PubMed: 21738725]

Arcelus J, \& Vostanis P (2005). Psychiatric comorbidity in children and adolescents. Current Opinion in Psychiatry, 18, 429-434. doi:10.1097/01.yco.0000172063.78649.66 [PubMed: 16639137]

Armour C, Fried EI, \& Olff M (2017). PTSD symptomics: network analyses in the field of psychotraumatology. European Journal of Psychotraumatology, 8:sup3, 1398003, doi:10.1080/20008198.2017.1398003 [PubMed: 29250305]

Babyak MA (2004). What you see may not be what you get: a brief, nontechnical introduction to overfitting in regression-type models. Psychosomatic Medicine, 66, 411-421. doi:10.1097/01.psy.0000127692.23278.a9 [PubMed: 15184705]

Bates D, \& Maechler M (2010). Matrix: sparse and dense matrix classes and methods. R package version 0.999375-43, http://cran.r-project.org/package=Matrix

Beard C, Millner AJ, Forgeard MJ, Fried EI, Hsu KJ, Treadway MT, ... \& Björgvinsson T (2016). Network analysis of depression and anxiety symptom relationships in a psychiatric sample. Psychological Medicine, 46, 3359-3369. doi:10.1017/S0033291716002300 [PubMed: 27623748]

Berg JM, Latzman RD, Bliwise NG, \& Lilienfeld SO (2015). Parsing the heterogeneity of impulsivity: A meta-analytic review of the behavioral implications of the UPPS for psychopathology. Psychological Assessment, 27, 1129-1146. doi:10.1037/pas0000111 [PubMed: 25822833]

Borsboom D, \& Cramer AO (2013). Network analysis: an integrative approach to the structure of psychopathology. Annual Review of Clinical Psychology, 9, 91-121. doi:10.1146/annurevclinpsy-050212-185608

Borsboom D (2017). A network theory of mental disorders. World Psychiatry, 16, 5-13. doi:10.1002/ wps.20375 [PubMed: 28127906]

Borsboom D, Fried EI, Epskamp S, Waldorp LJ, van Borkulo CD, van der Maas HLJ, Cramer AOJ (2017). False alarm? A comprehensive reanalysis of "Evidence that psychopathology symptom networks have limited replicability" by Forbes, Wright, Markon, and Krueger (2017). Journal of Abnormal Psychology, 126, 989-999. doi:10.1037/abn0000306 [PubMed: 29106282]

Boschloo L, van Borkulo CD, Rhemtulla M, Keyes KM, Borsboom D, \& Schoevers RA (2015). The network structure of symptoms of the diagnostic and statistical manual of mental disorders. PLoS One, 10, e0137621. doi:10.1371/journal.pone.0137621 [PubMed: 26368008]

Boschloo L, Schoevers RA, van Borkulo CD, Borsboom D, \& Oldehinkel AJ (2016). The network structure of psychopathology in a community sample of preadolescents. Journal of Abnormal Psychology, 125, 599-606. doi:10.1037/abn0000150 [PubMed: 27030994]

Briley DA, Harden KP, Bates TC, \& Tucker-Drob EM (2015). Nonparametric estimates of gene $\times$ environment interaction using local structural equation modeling. Behavior Genetics, 45, 581-596. doi:10.1007/s10519-015-9732-8 [PubMed: 26318287]

Carver CS, Johnson SL, \& Timpano KR (2017). Toward a Functional View of the p Factor in Psychopathology. Clinical Psychological Science, 5, 880-889. doi:10.1177/2167702617710037 [PubMed: 29057170]

Caspi A, Houts RM, Belsky DW, Goldman-Mellor SJ, Harrington H, Israel S, ... \& Moffitt TE (2014). The p factor: one general psychopathology factor in the structure of psychiatric disorders? Clinical Psychological Science, 2, 119-37. doi:10.1177/2167702613497473 [PubMed: 25360393]

Caspi A, \& Moffitt TE (2018). All for One and One for All: Mental Disorders in One Dimension. American Journal of Psychiatry, in press, appi-ajp. doi:10.1176/appi.ajp.2018.17121383

Chuderski A, Nęcka E (2010) Intelligence and Cognitive Control In: Gruszka A, Matthews G, Szymura B (eds) Handbook of Individual Differences in Cognition. The Springer Series on Human Exceptionality. Springer, New York, NY. doi:10.1007/978-1-4419-1210-7_16

Clark LA, Cuthbert B, Lewis-Fernández R, Narrow WE, \& Reed GM (2017). Three Approaches to Understanding and Classifying Mental Disorder: ICD-11, DSM-5, and the National Institute of 
Mental Health's Research Domain Criteria (RDoC). Psychological Science in the Public Interest, 18, 72-145. doi:10.1177/1529100617727266 [PubMed: 29211974]

Conners CK, Pitkanen J, \& Rzepa SR (2011). Conners comprehensive behavior rating scale In Encyclopedia of Clinical Neuropsychology (pp. 678-680). Springer New York.

Costantini G, \& Perugini M (2016). The network of conscientiousness. Journal of Research in Personality, 65, 68-88. doi:10.1016/j.jrp.2016.10.003

Cramer AO, Waldorp LJ, van der Maas HL, \& Borsboom D (2010). Complex realities require complex theories: Refining and extending the network approach to mental disorders. Behavioral and Brain Sciences, 33, 178-193. doi:10.1017/S0140525X1000092

Csardi G, \& Nepusz T (2006). The igraph software package for complex network research. Inter Journal, Complex Systems, 1695, 1-9. http://igraph.org

Curtiss J, \& Klemanski DH (2016). Taxonicity and network structure of generalized anxiety disorder and major depressive disorder: an admixture analysis and complex network analysis. Journal of Affective Disorders, 199, 99-105. doi:10.1016/j.jad/2016.04.007 [PubMed: 27100054]

Curtiss J, Ito M, Takebayashi Y, \& Hofmann SG (2018). Longitudinal network stability of the functional impairment of anxiety and depression. Clinical Psychological Science, 6, 325-334. doi: $10.1177 / 2167702617745640$

Cyders MA, \& Smith GT (2007). Mood-based rash action and its components: Positive and negative urgency. Personality and Individual Differences, 43, 839-850. doi:10.1016/j.paid.2007.02.008

Cyders MA, \& Smith GT (2010). Longitudinal validation of the urgency traits over the first year of college. Journal of Personality Assessment, 92, 63-69. doi: 10.1080/00223890903381825 [PubMed: 20013457]

David AS, Zammit S, Lewis G, Dalman C, \& Allebeck P (2008). Impairments in cognition across the spectrum of psychiatric disorders: evidence from a Swedish conscript cohort. Schizophrenia Bulletin, 34, 1035-1041. doi:10.1093/schbul/sbn028 [PubMed: 18441331]

Dirks MA, De Los Reyes A, Briggs-Gowan MJ, Cella D, \& Wakschlag LS (2012). Embracing not erasing contextual variability in children's behavior. Journal of Child Psychology and Psychiatry, 53, 558-574. doi:10.1111/j.1469-7610.2012.02537.x [PubMed: 22360546]

Ebesutani C, Bernstein A, Nakamura BJ, Chorpita BF, Higa-McMillan CK, Weisz JR, \& Research Network on Youth Mental Health. (2010). Concurrent validity of the Child Behavior Checklist DSM-oriented scales: Correspondence with DSM diagnoses and comparison to syndrome scales. Journal of Psychopathology and Behavioral Assessment, 32, 373-384. doi:10.1007/ s10862-009-9174-9 [PubMed: 20700377]

Engelhardt LE, Mann FD, Briley DA, Church JA, Harden KP, \& Tucker-Drob EM (2016). Strong genetic overlap between executive functions and intelligence. Journal of Experimental Psychology: General, 145, 1141-1159. doi:10.1037/xge0000195 [PubMed: 27359131]

Epskamp S, Cramer AO, Waldorp LJ, Schmittmann VD, \& Borsboom D (2012). qgraph: Network visualizations of relationships in psychometric data. Journal of Statistical Software, 48, 1-18. URL http://www.jstatsoft.org/v48/i04/.

Epskamp S, \& Fried EI (2016). A tutorial on regularized partial correlation networks. Arxiv Preprint (ID 1607.01367), 1-21. Retrieved from https://arxiv.org/abs/1607.01367

Epskamp S, Waldorp LJ, Mõttus R, \& Borsboom D (2018). The Gaussian Graphical Model in CrossSectional and Time-Series Data. Multivariate Behavioral Research, 1-28. doi: 10.1080/00273171.2018.1454823

Erford BT (1995). Reliability and Validity of the Conners Teacher Rating Scale-28 (CTRS-28). Diagnostique, 21, 19-28.

Fair DA, Bathula D, Nikolas MA, \& Nigg JT (2012). Distinct neuropsychological subgroups in typically developing youth inform heterogeneity in children with ADHD. Proceedings of the National Academy of Sciences, 109, 6769-6774. doi:10.1073/pnas.1115365109

Fisher AJ (2015). Toward a dynamic model of psychological assessment: Implications for personalized care. Journal of Consulting and Clinical Psychology, 83, 825-836. doi:10.1037/ccp0000026 [PubMed: 26009781] 
Fite PJ, Stoppelbein L, \& Greening L (2009). Proactive and reactive aggression in a child psychiatric inpatient population: Relations to psychopathic characteristics. Criminal Justice and Behavior, 36, 481-493. doi:10.1177/0093854809332706

Fite PJ, Rathert JL, Stoppelbein L, \& Greening L (2012). Social problems as a mediator of the link between reactive aggression and withdrawn/depressed symptoms. Journal of Child and Family Studies, 21, 184-189. doi:10.1007/s10826-011-9461-4

Fite PJ, Rubens SL, Preddy TM, Raine A, \& Pardini DA (2014). Reactive/proactive aggression and the development of internalizing problems in males: The moderating effect of parent and peer relationships. Aggressive Behavior, 40, 69-78. doi:10.1002/ab.21498 [PubMed: 23868672]

Fossati A, Gratz KL, Maffei C, \& Borroni S (2014). Impulsivity dimensions, emotion dysregulation, and borderline personality disorder features among Italian nonclinical adolescents. Borderline Personality Disorder and Emotion Dysregulation, 1, 5. doi: 10.1186/2051-6673-1-5 [PubMed: 26401289]

Foygel R, \& Drton M (2010). Extended Bayesian information criteria for Gaussian graphical models. In Advances in Neural Information Processing Systems, 604-612.

Fried EI, Boschloo L, van Borkulo CD, Schoevers RA, Romeijn J-W, Wichers MC, de Jonge P, Nesse RM, Tuerlinckx F, Borsboom D (2015). Symptomics as a new research paradigm for psychiatry. Frontiers in Psychiatry, 6, 1-3. doi:10.3389/fpsyt.2015.00117 [PubMed: 25653621]

Fried EI, \& Nesse RM (2015a). Depression is not a consistent syndrome: an investigation of unique symptom patterns in the STAR* D study. Journal of Affective Disorders, 172, 96-102. doi:10.1016/j.jad.2014.10.010 [PubMed: 25451401]

Fried EI, \& Nesse RM (2015b). Depression sum-scores don't add up: why analyzing specific depression symptoms is essential. BMC Medicine, 13, 1-11. doi:10.1186/s12916-015-0325-4 [PubMed: 25563062]

Fried EI, Epskamp S, Nesse RM, Tuerlinckx F, \& Borsboom D (2016). What are 'good' depression symptoms? Comparing the centrality of DSM and non-DSM symptoms of depression in a network analysis. Journal of Affective Disorders, 189, 314-320. doi:10.1016/j.jad.2015.09.005 [PubMed: 26458184]

Fried EI, van Borkulo CD, Cramer AO, Boschloo L, Schoevers RA, \& Borsboom D (2017). Mental disorders as networks of problems: a review of recent insights. Social Psychiatry and Psychiatric Epidemiology, 52, 1-10. doi:10.1007/s00127-016-1319-z [PubMed: 27921134]

Fried EI, Eidhof MB, Palic S, Costantini G, Huisman-van Dijk HM, Bockting CL, .. \& \& Karstoft KI (2018). Replicability and Generalizability of Posttraumatic Stress Disorder (PTSD) Networks: A Cross-Cultural Multisite Study of PTSD Symptoms in Four Trauma Patient Samples. Clinical Psychological Science, 6, 335-351. doi:10.1177/2167702617745092 [PubMed: 29881651]

Friedman JH, Hastie T, and Tibshirani R (2014). glasso: Graphical lasso-estimation of Gaussian graphical models. R package version $1.8 \mathrm{https} / /$ CRAN.R-project.org/package=glasso

Fruchterman TM, \& Reingold EM (1991). Graph drawing by force-directed placement. Software: Practice and Experience, 21, 1129-1164. doi: 10.1002/spe.4380211102

Goekoop R, \& Goekoop JG (2014). A network view on psychiatric disorders: network clusters of symptoms as elementary syndromes of psychopathology. PloS one, 9, e112734. doi:10.1371/ journal.pone.0112734 [PubMed: 25427156]

Graham JW (2009). Missing data analysis: Making it work in the real world. Annual Review of Psychology, 60, 549-576. doi:10.1146/annurev.psych.58.110405.085530.

Griffith JW, Zinbarg RE, Craske MG, Mineka S, Rose RD, Waters AM, \& Sutton JM (2010). Neuroticism as a common dimension in the internalizing disorders. Psychological Medicine, 40, 1125-1136. doi:10.1017/S0033291709991449 [PubMed: 19903363]

Harden KP, Tucker-Drob EM, \& Tackett JL (2013). The Texas twin project. Twin Research and Human Genetics, 16, 385-390. doi:10.1017/thg.2012.97 [PubMed: 23111007]

Harden KP, Engelhardt LE, Mann FD, Patterson MW, Grotzinger AD, Savicki SL..Tucker-Drob EM (2017). Overlapping genetic influences on executive functions, intelligence, and a general factor of psychopathology. Under review. 
Hasin D, \& Kilcoyne B (2012). Comorbidity of psychiatric and substance use disorders in the United States: current issues and findings from the NESARC. Current Opinion in Psychiatry, 25, 165171. doi:10.1097/YCO.0b013e3283523dcc [PubMed: 22449770]

Hettema JM, Neale MC, Myers JM, Prescott CA, \& Kendler KS (2006). A population-based twin study of the relationship between neuroticism and internalizing disorders. American Journal of Psychiatry, 163, 857-864. doi:10.1176/ajp.2006.163.5.857 [PubMed: 16648327]

Hildebrandt A, Wilhelm O, \& Robitzsch A (2009). Complementary and competing analytic approaches for the investigation of measurement invariance. Review of Psychology, 16, 87-102.

Hildebrandt A, Luedtke O, Robitzsch A, Sommer C, \& Wilhelm O (2016). Exploring factor model parameters across continuous variables with local structural equation models. Multivariate Behavioral Research, 51, 257-278. doi: 10.1080/00273171.2016.1142856 [PubMed: 27049892]

Hofmann SG, Curtiss J, \& McNally RJ (2016). A complex network perspective on clinical science. Perspectives on Psychological Science, 11, 597-605. doi:10.1177/1745691616639283 [PubMed: 27694457]

Hunsley J, \& Mash EJ (2007). Evidence-based assessment. Annual Review of Clinical Psychology, 3, 29-51. doi:10.1146/annurev.clinpsy.3.022806.091419

John OP, \& Srivastava S (1999). The Big Five trait taxonomy: History, measurement, and theoretical perspectives. Handbook of Personality: Theory and Research, 2, 102-138.

Johnson SL, Carver CS, \& Joormann J (2013). Impulsive responses to emotion as a transdiagnostic vulnerability to internalizing and externalizing symptoms. Journal of Affective Disorders, 150, 872-878. doi:10.1016/j.jad.2013.05.004 [PubMed: 23726781]

Johnson SL, Tharp JA, Peckham AD, Carver CS, \& Haase CM (2017). A path model of different forms of impulsivity with externalizing and internalizing psychopathology: towards greater specificity. British Journal of Clinical Psychology, 56, 235-252. doi:10.1111/bjc.12135 [PubMed: 28493489]

Jones P (2018). networktools: Tools for Identifying Important Nodes in Networks. R package version 1.1.1 https://CRAN.R-project.org/package=networktools

Kessler RC, Chiu WT, Demler O, \& Walters EE (2005a). Prevalence, severity, and comorbidity of 12month DSM-IV disorders in the National Comorbidity Survey Replication. Archives of General Psychiatry, 62, 617-627. doi:10.1001/archpsyc.62.6.617 [PubMed: 15939839]

Kessler RC, Berglund P, Demler O, Jin R, Merikangas KR, \& Walters EE (2005b). Lifetime prevalence and age-of-onset distributions of DSM-IV disorders in the National Comorbidity Survey Replication. Archives of General Psychiatry, 62, 593-602. doi:10.1001/archpsyc.62.6.593 [PubMed: 15939837]

Koechlin E, Ody C, \& Kouneiher F (2003). The architecture of cognitive control in the human prefrontal cortex. Science, 302, 1181-1185. doi:10.1126/science.1088545 [PubMed: 14615530]

Koenen KC, Moffitt TE, Roberts AL, Martin LT, Kubzansky L, Harrington H, ... \& Caspi A (2009). Childhood IQ and adult mental disorders: a test of the cognitive reserve hypothesis. American Journal of Psychiatry, 166, 50-57. doi:10.1176/appi.ajp.2008.08030343 [PubMed: 19047325]

Kret ME, \& Ploeger A (2015). Emotion processing deficits: a liability spectrum providing insight into comorbidity of mental disorders. Neuroscience \& Biobehavioral Reviews, 52, 153-171. doi:10.1016/j.neubiorev.2015.02.01 [PubMed: 25725415]

Kring AM, \& Sloan DM (Eds.). (2009). Emotion regulation and psychopathology: A transdiagnostic approach to etiology and treatment. Guilford Press.

Krueger RF, Hicks BM, Patrick CJ, Carlson SR, Iacono WG, \& McGue M (2002). Etiologic connections among substance dependence, antisocial behavior and personality: Modeling the externalizing spectrum. Journal of Abnormal Psychology, 111, 411-424. doi:10.1037//0021-843X.111.3.411 [PubMed: 12150417]

Krueger RF, Markon KE, Patrick CJ, \& Iacono WG (2005). Externalizing psychopathology in adulthood: A dimensional-spectrum conceptualization and its implications for DSM-V. Journal of Abnormal Psychology, 114, 537-550. doi:10.1037/0021-843X.114.4.537 [PubMed: 16351376]

Lahey BB, Rathouz PJ, Keenan K, Stepp SD, Loeber R, \& Hipwell AE (2015). Criterion validity of the general factor of psychopathology in a prospective study of girls. Journal of Child Psychology and Psychiatry, 56, 415-422. doi: 10.1111/jcpp.12300 [PubMed: 25052460] 
Lindhiem O, Bennett CB, Hipwell AE, \& Pardini DA (2015). Beyond symptom counts for diagnosing oppositional defiant disorder and conduct disorder? Journal of Abnormal Child Psychology, 43, 1379-1387. doi:10.1007/s10802-015-0007-x [PubMed: 25788042]

Lundervold AJ, Bøe T, \& Lundervold A (2017). Inattention in primary school is not good for your future school achievement-A pattern classification study. PloS one, 12, e0188310. doi:10.1371/ journal.pone.0188310 [PubMed: 29182663]

Marmorstein NR (2013). Associations between dispositions to rash action and internalizing and externalizing symptoms in children. Journal of Clinical Child \& Adolescent Psychology, 42, 131138. doi:10.1080/15374416.2012.734021 [PubMed: 23095038]

McNally RJ (2016). Can network analysis transform psychopathology? Behaviour Research and Therapy, 86, 95-104. doi:10.1016/j.brat.2016.06.006 [PubMed: 27424882]

Michaud PA, \& Fombonne E (2005). Common mental health problems. BMJ, 330, 835-838. doi:10.1136/bmj.330.7495.835 [PubMed: 15817553]

Muthén LK, \& Muthen BO (1998-2011). Mplus User's Guide. Sixth Edition. Los Angeles, CA: Muthén \& Muthén.

Nakamura BJ, Ebesutani C, Bernstein A, \& Chorpita BF (2009). A psychometric analysis of the child behavior checklist DSM-oriented scales. Journal of Psychopathology and Behavioral Assessment, 31, 178-189. doi:10.1007/s10862-008-9119-8

Neumann A, Pappa I, Lahey BB, Verhulst FC, Medina-Gomez C, Jaddoe VW, ... \& Tiemeier H (2016). Single nucleotide polymorphism heritability of a general psychopathology factor in children. Journal of the American Academy of Child \& Adolescent Psychiatry, 55, 1038-1045. doi:10.1016/j.jaac.2016.09.498 [PubMed: 27871638]

Reichardt J, \& Bornholdt S (2006). Statistical mechanics of community detection. Physical Review E, 74(1), 016110 http://arxiv.org/abs/cond-mat/0603718

Rhemtulla M, Fried EI, Aggen SH, Tuerlinckx F, Kendler KS, Borsboom D (2016). Network analysis of substance abuse and dependence symptoms. Drug and Alcohol Dependence, 161, 230-237. doi:10.1016/j.drugalcdep.2016.02.005 [PubMed: 26898186]

Rigdon EE (1997, 6 11). Not positive definite matrices - causes and cures. Retrieved from http:// www2.gsu.edu/ mkteer/npdmatri.html

Robinaugh DJ, Leblanc NJ, Vuletich HA, McNally RJ (2014). Network analysis of persistent complex bereavement disorder in conjugally bereaved adults. Journal of Abnormal Psychology, 123, 510522. doi:10.1037/abn0000002 [PubMed: 24933281]

Roiser JP, \& Sahakian BJ (2013). Hot and cold cognition in depression. CNS Spectrums, 18, 139-149. doi:10.1017/S1092852913000072 [PubMed: 23481353]

Rouquette A, Pingault JB, Fried EI, Orri M, Falissard B, Kossakowski JJ, ... \& Borsboom D (2018). Emotional and behavioral symptom network structure in elementary school girls and association with anxiety disorders and depression in adolescence and early adulthood: a network analysis. JAMA Psychiatry. Published online 8 15, 2018. doi:10.1001/jamapsychiatry.2018.2119

Rudolph KD, Lansford JE, \& Rodkin PC (2016). Interpersonal theories of developmental psychopathology. Developmental Psychopathology. doi:10.1002/9781119125556.devpsy307

Ruzzano L, Borsboom D, \& Geurts HM (2015). Repetitive behaviors in autism and obsessivecompulsive disorder: new perspectives from a network analysis. Journal of Autism and Developmental Disorders, 45, 192-202. doi:10.1007/s10803-014-2204-9 [PubMed: 25149176]

Sijtsema JJ, Verboom CE, Penninx BWJH, Verhulst FC, \& Ormel J (2014). Psychopathology and academic performance, social well-being, and social preference at school: the TRAILS study. Child Psychiatry \& Human Development, 45, 273-284. doi:10.1007/s10578-013-0399-1 [PubMed: 23917997]

Smith GT, Guller L, \& Zapolski TC (2013). A comparison of two models of urgency: Urgency predicts both rash action and depression in youth. Clinical Psychological Science, 1, 266-275. doi:10.1177/2167702612470647 [PubMed: 25419495]

Snyder HR, Miyake A, \& Hankin BL (2015). Advancing understanding of executive function impairments and psychopathology: bridging the gap between clinical and cognitive approaches. Frontiers in Psychology, 6, 328. doi:10.3389/fpsyg.2015.00328 [PubMed: 25859234] 
Solomon M, Ozonoff SJ, Cummings N, \& Carter CS (2008). Cognitive control in autism spectrum disorders. International Journal of Developmental Neuroscience, 26, 239-247. doi:10.1016/ j.ijdevneu.2007.11.001 [PubMed: 18093787]

Svaldi J, Griepenstroh J, Tuschen-Caffier B, \& Ehring T (2012). Emotion regulation deficits in eating disorders: a marker of eating pathology or general psychopathology? Psychiatry Research, 197, 103-111. doi:10.1016/j.psychres.2011.11.009 [PubMed: 22401969]

Van Buuren S, Groothuis-Oudshoorn K (2011) mice: Multivariate Imputation by Chained Equations in R. Journal of Statistical Software, 45 URL http://www.jstatsoft.org/v45/i03/.

Wechsler D (2011). Wechsler Abbreviated Scale of Intelligence-2nd ed. (WASI-II). San Antonio, TX: NCS Pearson.

Werner K, \& Gross JJ (2010). Emotion regulation and psychopathology: A conceptual framework In Kring AM \& Sloan DM (Eds.), Emotion regulation and psychopathology: A transdiagnostic approach to etiology and treatment (pp. 13-37). New York, NY, US: Guilford Press.

Whiteside SP, \& Lynam DR (2001). The five factor model and impulsivity: Using a structural model of personality to understand impulsivity. Personality and Individual Differences, 30, 669-689. doi:10.1016/S0191-8869(00)00064-7

Whiteside SP, Lynam DR, Miller JD, \& Reynolds SK (2005). Validation of the UPPS impulsive behaviour scale: a four-factor model of impulsivity. European Journal of Personality, 19, 559574. doi:10.1002/per.556

Wright AG, Hallquist MN, Morse JQ, Scott LN, Stepp SD, Nolf KA, \& Pilkonis PA (2013). Clarifying interpersonal heterogeneity in borderline personality disorder using latent mixture modeling. Journal of Personality Disorders, 27, 125-143. doi:10.1521/pedi.2013.27.2.125 [PubMed: 23514179]

Zapolski TC, Stairs AM, Settles RF, Combs JL, \& Smith GT (2010). The measurement of dispositions to rash action in children. Assessment, 17, 116-125. doi: 10.1177/1073191109351372 [PubMed: 19955108]

Zelazo PD, \& Müller U (2002). Executive function in typical and atypical development. Blackwell Handbook of Childhood Cognitive Development, 445-469. doi:10.1002/9780470996652.ch20 


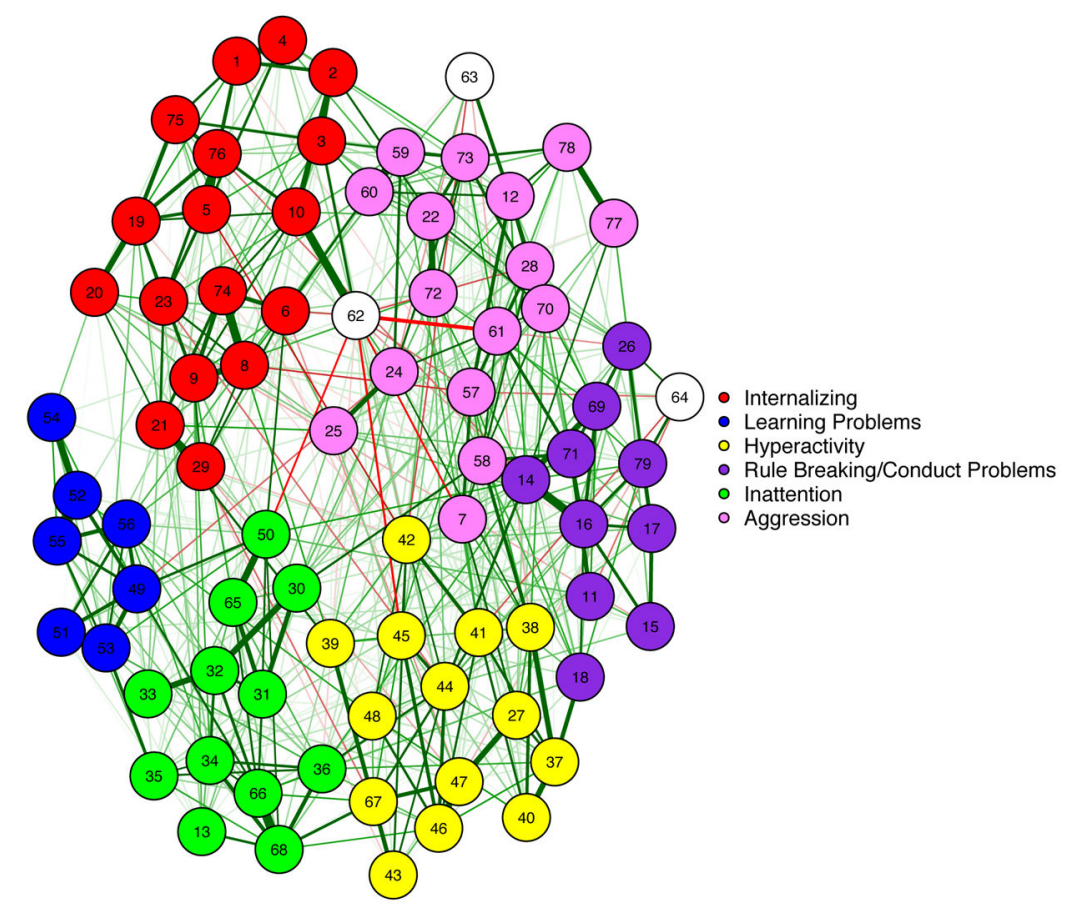

Figure 1.

Network of 76 psychopathology items, controlling for age, gender, and ethnicity (white nodes). Items are represented as nodes and associations between them as edges. Green edges indicate positive associations and red edges indicate negative associations, with the thickness of the edge indicating the strength of the association. Groups are derived from the spinglass algorithm, which maximizes between cluster separation and within cluster cohesion based on the number of connections nodes share (Reichardt \& Bornholdt, 2006). Groups are labeled based on the self-report subscale the majority of the items in the cluster come from. 


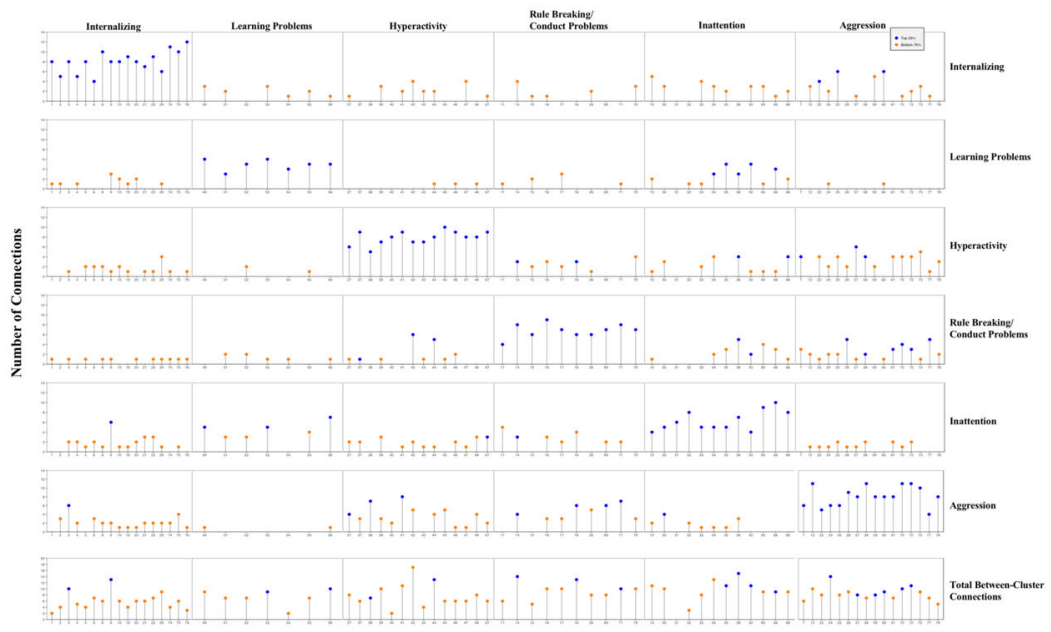

Figure 2.

Number of connections from each individual symptom to each domain of problem behaviors. Horizontal panels represent the domains within the network, where the x-axis of each panel represents each node within the network, grouped by domain. The y-axis of each panel represents the number of connections that each node displays with the respective domain. The last panel represents the number and strength of each node's connections to all nodes outside of its domain. Connections are colored based on the average edge weight of each node to each domain (i.e., all nodes within a particular domain). For the six individual domain panels, if the average node to domain edge weight was greater than .0116 (75th percentile and above) the node was colored blue (i.e., "relatively strong"). Connections for each node to all five between-cluster domains are colored based on the average edge weight from an individual node to all out-of-cluster nodes. For the total between-cluster connections, if the grand average node to domain edge weight was greater than .0262 (75th percentile and above), the node was colored blue. 

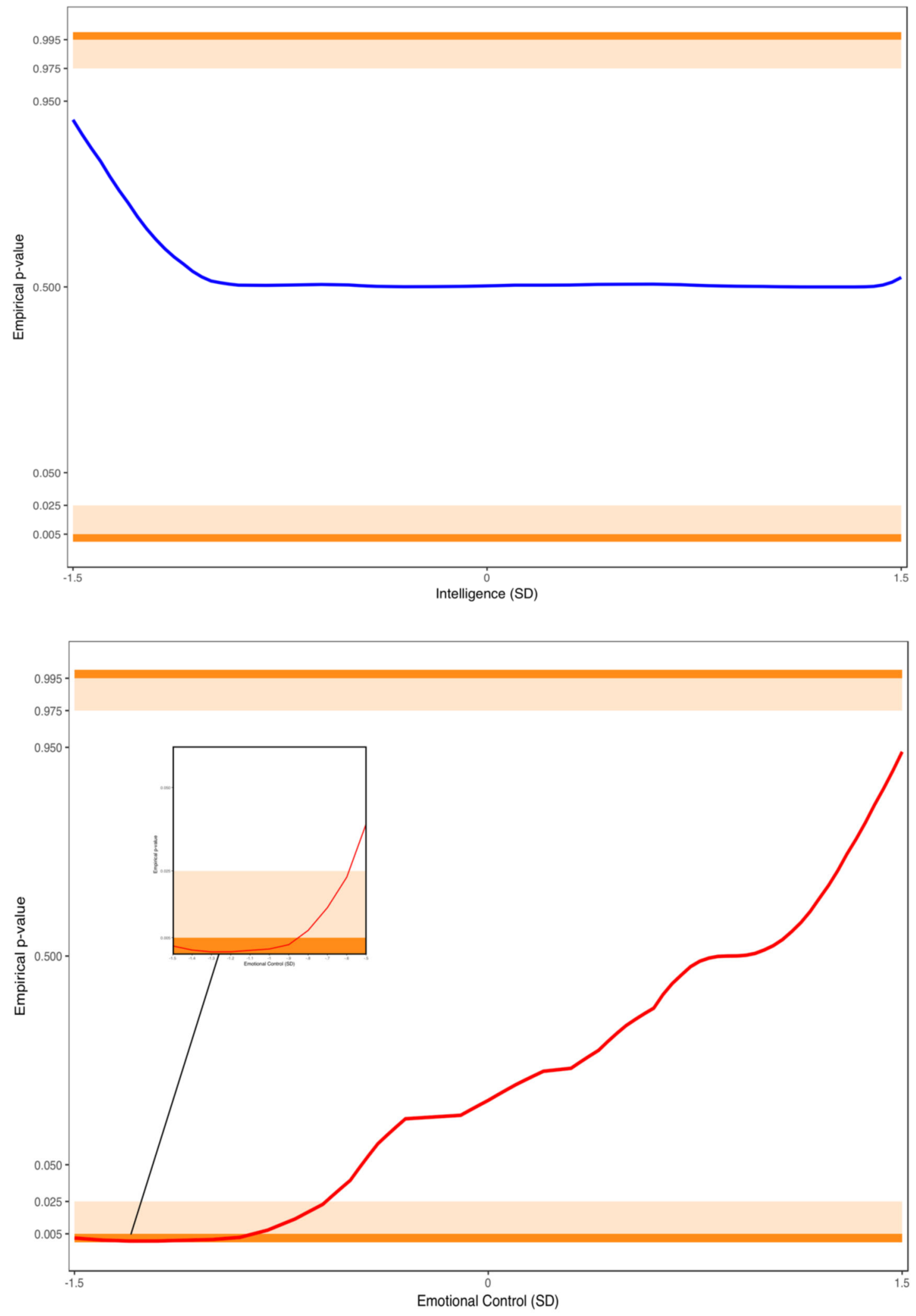

Figure 3.

Clin Psychol Sci. Author manuscript; available in PMC 2020 April 17. 
The average between-cluster edge weight $(\mathrm{Mr}(\mathrm{BC}))$ in 31 LOSEM-weighted networks across the range of intelligence (blue line) and emotional control (red line). Orange bands indicate empirical $\mathrm{p}$-value thresholds $(\mathrm{p}<.025 ; \mathrm{p}<.005)$ at both tails of the distribution. Empirical p-values were generated by comparing observed $\operatorname{Mr}(\mathrm{BC})$ values to the empirical null distribution. 